\title{
BARYON STOPPING AND HYPERON ENHANCEMENT IN THE IMPROVED DUAL PARTON MODEL
}

\author{
A. Capella and C. A. Salgado \\ Laboratoire de Physique Théorique $\mathrm{f}$ \\ Université de Paris XI, Bâtiment 210, F-91405 Orsay Cedex, France
}

\begin{abstract}
We present an improved version of the dual parton model which contains a new realization of the diquark breaking mechanism of baryon stopping. We reproduce in this way the net baryon yield in nuclear collisions. The model, which also considers strings originating from diquark-antidiquark pairs in the nucleon sea, reproduces the observed yields of $p$ and $\Lambda$ and their antiparticles and underestimates cascades by less than $50 \%$. However, $\Omega$ 's are underestimated by a factor five. Agreement with data is restored by final state interaction, with an averaged cross-section as small as $\sigma=0.14 \mathrm{mb}$. Hyperon yields increase significantly faster than antihyperons, in agreement with experiment.
\end{abstract}

LPT Orsay 99-20

March 1999

*e-mail : capella@qcd.th.u-psud.fr

${ }^{\dagger}$ e-mail : salgado@qcd.th.u-psud.fr

†Unité Mixte de Recherche (CNRS) UMR 8627 


\section{Introduction}

A striking feature of heavy ion collisions is the huge stopping of the participating nucleons. At CERN energies, the deep minimum in the net baryon rapidity distribution $(\Delta B=B-\bar{B})$ at $y^{*} \sim 0$, observed in $p p$ collisions, has been practically filled up in a central collision of heavy ions [1, 2]. For central $\mathrm{Pb}-\mathrm{Pb}$ collisions, the value of this density is five times larger than the corresponding value in $p p-$ scaled by the average number of participants. Note that the total number of $B-\bar{B}$ (i.e. integrated over rapidity), exactly satisfies scaling in the number of participants, due to baryon number conservation. This shows the dramatic change in the shape of the $B-\bar{B}$ (and $p-\bar{p}$ ) rapidity distributions between $p p$ and central $P b-P b$ collisions. Such a change is usually referred to as baryon stopping.

All independent string models of hadronic and nuclear collisions in their original form completely fail to reproduce this important feature of heavy ion collisions. In the dual parton model (DPM) [3] and in the quark gluon string model (QGSM) [1], the dominant contribution to particle production in $p p$ collisions at $\sqrt{s} \sim 20 \mathrm{GeV}$, consists in two $q q-q$ strings, which produce, after fragmentation, two baryons in the fragmentation regions of the colliding protons. Starting with the Lund model, which initially had a single string, the above mechanism of particle production has been adopted in most current string models. In these models, there is some amount of stopping due to energy conservation. This produces an increase of the net baryon yield at mid rapidities between $\mathrm{NN}$ and central $\mathrm{Pb}-\mathrm{Pb}$ collisions, which is typically

of a factor 2 [3] - more than two times smaller than the observed one. Hence, the dramatic failure of all these models to reproduce the observed stopping.

Actually, a possibility to slow down the net baryon in $p p$ collisions, was introduced a long time ago by Rossi and Veneziano [5]. In their approach, the baryon is viewed as three valence quarks bound together by three strings each one with a quark at one end and with the other end joining in a point called string junction. This 
string junction carries momentum as well as the baryon quantum number. Rossi and Veneziano pointed out that the string junction could migrate to mid rapidities with a distribution in $d \sigma / d x \sim 1 / \sqrt{x}$ (or $d \sigma / d y \sim \exp \left(-1 / 2 \mid\left(y-y_{\max } \mid\right)\right.$. This corresponds to an annihilation cross-section which decreases with energy like $s^{-1 / 2}$. In refs. [6] and [7] a distribution of the string junction in $x^{-1}$ (i.e. flat in rapidity) was proposed, corresponding to an annihilation cross-section which reaches a constant asymptotic value (of 1 to $2 \mathrm{mb}$ ). Here we adopt the first approach. However, we do not rule out the second possibility - which would have important consequences at the energies of the future heavy ion colliders [8].

The above stopping mechanism has been recently introduced in heavy ion collisions [8] [9] and implemented in the Hijing [10] and Venus [11] Monte Carlos. However, the introduction of the Rossi-Veneziano mechanism does not explain by itself why the stopping is larger in central heavy ion collisions than in $p p$. In ref. [8], a mechanism to enhance stopping in heavy ion collisions was proposed. It was based on the separation of the $p p$ cross-section $\sigma_{p p}=\sigma_{p p}^{D P}+\sigma_{p p}^{D B}$ into a diquark breaking $(D B)$ and a diquark preserving $(D P)$ piece, and on the assumption that the diquark can be broken in any inelastic collision. These results in a $D B$ cross section in $p A$ and $A A$ collisions which increases faster with $A$ than the $D P$ one. The drawback of this approach is that it requires some fine tuning. The value of $\sigma_{p p}^{D B}$ has to be small enough in order not to contradict the $p p$ and $p A$ data (where stopping is comparatively small) and large enough to produce the large stopping observed in central heavy ion collisions.

In a recent publication [12], a new formulation of the $D B$ mechanism has been introduced in which this drawback is avoided (i.e. one can have $\sigma_{p p}^{D B}$ negligeably small at CERN energies, and still have an important effect in central $\mathrm{Pb}-\mathrm{Pb}$ collisions). In the present paper we use the formulation of [12 to compute the rapidity distributions of $B-\bar{B}$ in hadronic and nuclear collisions. We obtain a reasonable 
agreement with experiment[

Another striking feature of the CERN heavy ion program is the strong increase of the yields of hyperons and anti-hyperons per participant between $p p$ or $p A$ and central $A B$ collisions. This increase obeys to the hierarchy $\Omega>\Xi>\Lambda$ (i.e. the large number of strange quarks in $Y$, the larger the increase) [13, 14]. In two recent publications [12] [15], it has been shown that a baryon stopping mechanism of the type described above produces a substantial increase of the hyperon yields according to this hierarchy. The physical reason for this increase is quite obvious [16]. Since in the diquark breaking component the net baryon is formed out of three sea quarks around the string junction, the probability of producing hyperons is strongly enhanced - especially for $\Omega$ 's since its probability of production in the conventional diquark fragmentation mechanism is zero. In the present paper we extend the results of [12] in two directions. First, we study the rapidity distributions of protons and hyperons in $p A$ and $A A$ collisions (in refs. [12] and [15] the analysis was restricted to the rapidity window $\left.\left|y^{*}\right|<0.5\right)$. Second, we show how the four free parameters of ref. [12] can actually be reduced to two. This makes the model more predictive, especially for the antihyperon over hyperon ratios.

While the yield of $\Lambda$ 's and, to a large extent, of cascades can be described by the model, that of omegas is underestimated by almost an order of magnitude. The same conclusion has been reached in [12, 15]. We show that final state interaction, with an averaged cross-section as small as $\sigma=0.14 \mathrm{mb}$ [12], allows to describe all hyperon and antihyperon yields.

A similar value of $\sigma$ was found in ref. [17] in the hadron gas model. It was argued there that, due to this small value of $\sigma$, interactions in a hadron gas could not drive the system to chemical equilibrium (the process would be too slow). We find, indeed, that the effect of final state interaction in $p$ and $\Lambda$ production is very

\footnotetext{
${ }^{\S}$ Note, however, that similar results are obtained using the approach of ref. [8] with the $D B$ mechanism of Fig. 2.
} 
small. Its effect on $\Xi$ production is moderate. Only for such a rare process as $\Omega$ production its effect is very important - making the $\Omega+\bar{\Omega}$ yield five times larger than the value obtained without final state interaction.

Due to the diquark breaking component, we obtain an increase of hyperons substantially larger than the one of antihyperons, i.e. the ratio between $\mathrm{Pb}-\mathrm{Pb}$ and $p P b$ yields is substantially larger for $Y$ than for $\bar{Y}$. This effect is enhanced by final state interaction. It has been observed experimentally [14.

The paper is organized as follows. In Section 2 we describe the baryon stopping mechanism and compute the net baryon $(B-\bar{B})$ rapidity distributions in $p p, p P b$, $S S$ and $P b-P b$ collisions. In Section 3 we compute the rapidity distributions of $p-\bar{p}$, and $Y-\bar{Y}$. In Section 4 we describe $B \bar{B}$ pair production from strings containing sea diquarks or antidiquarks at one of their ends and show how the $A$-dependence of $\bar{B}$ production is increased. In Section 5 we study the effect of the final state interaction, separately on the $Y$ and $\bar{Y}$ yields. Section 6 contains a discussion of our results. Conclusions are given in Section 7.

\section{$2 \quad$ Baryon stopping}

A fragmentation string mechanism in which the $x \rightarrow 0$ and $x \rightarrow 1$ behaviour of the fragmentation functions is controlled by Regge intercepts has been introduced in ref. [18]. In the case of net baryon production, it consists of a sum of two terms as depicted in Fig. 1. In the original Lund fragmentation scheme 19 only the first term is considered. The second one was introduced later - the so-called pop-corn mechanism. Even with the inclusion of the second component (Fig. 1b), this mechanism leads to the production of too fast baryons and fails completely to reproduce the observed stopping in heavy ion collisions. This fragmentation scheme (including the component in Fig. 1b) will be referred to as the conventional or diquark preserving $(D P)$ mechanism. Following ref. [12] we introduce the baryon 
stopping mechanism showed in Fig. 2. It will be referred to as the diquark breaking $(D B)$ component. In this component, the rapidity distribution of the produced net baryon $\Delta B=B-\bar{B}$ in a $N-N$ collision is

$$
\frac{d N_{D B}^{\Delta B}}{d y}(y)=C_{n_{1}, n_{2}}\left[Z_{+}^{1 / 2}\left(1-Z_{+}\right)^{n_{1}-3 / 2}+Z_{-}^{1 / 2}\left(1-Z_{-}\right)^{n_{2}-3 / 2}\right]
$$

where $Z_{ \pm}=\exp \left( \pm y-y_{\max }\right), n_{1}$ and $n_{2}$ are the average number of collisions suffered by the two colliding nucleons and $C_{n_{1}, n_{2}}$ is determined from the normalization to two. The factor $Z^{1 / 2}$ has already been discussed in the Introduction. The factor $(1-Z)$ gives the behavior near $y=y_{\max }$. There is some uncertainty concerning its power [8]. The value in eq. (1) is obtained as follows. From Fig. 2 we see that in order to produce the baryon at $y \sim y_{\max }$ it is necessary to slow down three quarks. Assuming they behave as $1 / \sqrt{x}$ at the energies under consideration 《4, we obtain a power $1 / 2$ for the case of Fig. 2 - which corresponds to $n=2$. In the general case of $n$ inelastic collisions we obtain the power $n-3 / 2$ in eq. (1).

The corresponding distribution in $A A$ collisions is 12

$$
\frac{d N^{A A \rightarrow \Delta B}}{d y}(y)=\frac{\bar{n}_{A}}{\bar{n}}\left[\bar{n}_{A}\left(\frac{d N_{D P}^{\Delta B}}{d y}(y)\right)_{\bar{n} / \bar{n}_{A}}+\left(\bar{n}-\bar{n}_{A}\right)\left(\frac{d N_{D B}^{\Delta B}}{d y}(y)\right)_{\bar{n} / \bar{n}_{A}}\right]
$$

Here $\bar{n}_{A}$ and $\bar{n}$ are the average number of participants of nucleus $A$ and the average number of collisions, respectively. $d N_{D P} / d y$ is given by the conventional, diquark preserving, hadronization mechanism for which we use the results of ref. [20], and $d N_{D B} / d y$ is given by eq. (1). The integral over $y$ of both rapidity distributions is equal to two (baryon number conservation).

Let us discuss the physical meaning of eq. (2) in $p p$ interactions $\left(\bar{n}_{A}=1\right)$. We see that in the case of a single inelastic collision $(\bar{n}=1)$, we recover the conventional $D P$ mechanism. The underlying assumption is that, in this case, the string junction follows the valence diquark and baryon production takes place in the conventional 
way. (We do not exclude a small admixture of the $D B$ component in this case, but experimental data do not require its presence). Consider next the case of two inelastic collisions $(\bar{n}=2)$. Here the underlying assumption is that there is an equal probability $(1 / 2)$ for the net baryon to be produced in any of the two collisions. However, in only one of them can the string junction follow the valence diquark and fragment in the conventional $(D P)$ way. In the other one, the string junction is free and baryon production takes place according to the $D B$ mechanism. The latter is responsible of (most of) the observed baryon stopping. The generalization to $\bar{n}$ inelastic collisions and to $A A$ interactions (eq. (2)) is then straightforward.

It is quite remarkable that such a simple mechanism, with no free parameter (modulo the uncertainty in the power of $1-Z$ in eq. (1), discussed above) gives a good description of the present data on the net baryon rapidity distribution.

Note that in the case of $p p$ interactions at $\sqrt{s} \sim 20 \mathrm{GeV}$, the two string component $(\bar{n}=1)$ dominates, and, as discussed above, we recover the usual $D P$ results. With increasing energies, the components with $\bar{n} \neq 1$ become increasingly important and baryon stopping will increase. Eq. (11) (with $\bar{n}_{A}=1$ ) not only gives definite predictions concerning this increase but, moreover, leads to specific qualitative features. In particular stopping will strongly depend on the charged particle multiplicity. A low multiplicity event sample selects low values of $\bar{n}$ where stopping will be comparatively small, while, at large multiplicities, stopping will be larger. Such a feature has been observed recently at HERA [21] - and discussed in ref. 222 in a different theoretical framework.

We turn next to the generalization of eq. (2) to asymmetric interactions such as $p A$. In this case one has

$$
\frac{d N^{p A \rightarrow \Delta B}}{d y}(y)=\frac{d N_{D P}^{q q^{A}-q_{v}^{p}}}{d y}(y)+(\bar{n}-1) \frac{d N_{D P}^{q q^{A}-q_{s}^{p}}}{d y}(y)+
$$




$$
+\frac{1}{\bar{n}}\left[\frac{d N_{D P}^{q_{v}^{A}-q q^{p}}}{d y}(y)+(\bar{n}-1) \frac{d N_{D B}^{q_{v}^{A}-q q_{p}}}{d y}(y)\right] .
$$

Here $d N^{q q^{A}-q_{v}^{p}\left(q_{s}^{p}\right)} / d y$ denotes the rapidity distribution of a string stretched between a diquark of one of the $\bar{n}$ wounded nucleons of $A$ and a valence (or sea) quark of the proton. (It is computed in DPM as a convolution of momentum distribution functions and fragmentation functions). Since each of the wounded nucleons suffers a single inelastic collision, only the $D P$ component is involved - with each diquark fragmenting in the nucleus fragmentation region $\left(y^{*}<0\right)$. The terms in the bracket correspond to the fragmentation of the incoming proton. Since it suffers $\bar{n}$ inelastic collisions, we have the $D P$ hadronization mechanism (with probability $1 / \bar{n}$ ) and the $D B$ one (with probability $(\bar{n}-1) / \bar{n})$. The latter is now given by the first term of eq. (四). All rapidity distributions, integrated over $y$, are equal to one in this case.

The $B-\bar{B}$ rapidity distributions obtained from eq. (2) in central $S S$ and $P b-P b$ collisions are shown in Fig. 3. Note that at mid-rapidities, these distributions are dominated by the $D B$ component. Not only the latter is proportional to $\bar{n}-\bar{n}_{A}$ (eq. (2)), but, moreover, $d N_{D B} / d y$ is larger than $d N_{D P} / d y$ at mid rapidities (see Table 1). Nevertheless, the existence of the two huge maxima of the $D P$ component in the fragmentation regions, still shows up in the $A A$ distribution. For a given system, the detailed shape of the $B-\bar{B}$ rapidity distribution depends on the power of $(1-Z)$ in eq. (11). As discussed above there is some theoretical uncertainty in the value of this power. However, the variation of the shape of this rapidity distribution from one system to another is a characteristic feature of the model. As seen in Fig. 3, the minimum at mid-rapidities is gradually filled up from $p p$ to central $P b-P b$ collisions and, therefore, it is more pronounced in $S S$ than in $P b-P b$. 


\section{Net hyperon enhancement}

In the previous section we have shown that baryon stopping can be described using a new formulation of the diquark breaking $(D B)$ mechanism. In this case, depicted in Fig. 2, the string junction is surrounded by three sea quarks to produce the net baryon. Therefore, not only the net proton yield $\Delta p=p-\bar{p}$ will be strongly enhanced from $p p$ to central $A A$ collisions, but also the net hyperon yield $\Delta Y=$ $Y-\bar{Y}$. This is especially so for $\Omega$ 's, which cannot be produced at all with the $D P$ mechanism of Figs. 1a and $1 \mathrm{~b}$. More precisely the ratio between yields in central $A A$ and $p A$ (or $p p)$ collisions will obey to the hierarchy : $\Delta \Omega>\Delta \Xi>\Delta \Lambda>\Delta p$. This is in agreement with the results of the WA97 [14] and NA49 [1], 13] collaborations.

The relative yields of the different baryon species will be determined by the strangeness suppression factor $S / L$ where $S$ is the probability associated to the strange quark and $L$ the one associated to the light quarks ( $u$ or $d$ ). We consider two possibilities : $S=0.10$ and $L=(1-S) / 2=0.45(S / L=0.22)$ and $S=0.13$ and $L=0.435(S / L=0.3)$. With baryons produced out of three sea quarks (Fig. 2) it is easy to see that the relative yields are

$$
I_{3}=4 L^{3}: 4 L^{3}: 12 L^{2} S: 3 L S^{2}: 3 L S^{2}: S^{3}
$$

for $p, n, \Lambda+\Sigma, \Xi^{0}, \Xi^{-}$and $\Omega$, respectively. Moreover, we take, $\Sigma^{+}+\Sigma^{-}=0.6 \Lambda$. This reduction in the number of charged $\Sigma$ 's is due to resonance decay $\left(\Sigma(1385) P_{13}\right.$ decays into $\Lambda \pi$ with an $88 \pm 2 \%$ fraction).

It is interesting that, in spite of the huge hyperon enhancement observed experimentally, the factors (4) lead (both with $S=0.10$ and $S=0.13$ ) to an overestimation of hyperon production in $p P b$ collisions especially for $\Xi$ 's and $\Omega$ 's. In central $P b-P b$ collisions, net hyperon production is also overestimated - except for $\Omega$ 's. In ref. 12

\footnotetext{
I A new component consisting in a diquark which contains sea quarks has been introduced in 23. However, this diquark is assumed to have the same momentum distribution as a diquark made out of two valence quarks and, hence, produces baryons mainly in the fragmentation regions.
} 
the following explanation of this hyperon excess was proposed: at present energies, it may happen that the net baryon is not formed out of three sea quarks as in Fig. 2, but, due to phase space limitation, a valence quark, at one of the ends of the string where the baryon is produced, is picked up together with two sea quarks. Obviously, in this case the strangeness production rate is substantially reduced (in particular, $\Omega$ production is not possible in this case). The relative yields $I_{3}$ in (田) are then changed into

$$
I_{2}=2 L^{2}: 2 L^{2}: 4 L S: S^{2} / 2: S^{2} / 2: 0
$$

In the following, we introduce a free parameter $\alpha(0<\alpha<1)$ which determines the admixture of $I_{3}$ and $I_{2}$ given by (丑) and (5). More precisely, we will take the relative yields given by

$$
I=\alpha I_{3}+(\alpha-1) I_{2}
$$

The best description of the data is obtained with $\alpha=0.23$ for $S=0.13$ and $\alpha=0.5$ for $S=0.1$. The results are similar in the two cases; the sensitivity to the value of the strangeness suppression factor $S / L$ turns out to be quite small. The results for the rapidity distribution of the net yields of $p$ and $\Lambda$ in central $P b-P b$ collisions are given in Figs. 4 and 5. Note that our rapidity distribution for $\Lambda-\bar{\Lambda}$ (Fig. 5) is broader than the estimates of the NA49 collaboration [1]. This, in turn, produces some discrepancies in the $p-\bar{p}$ yield (Fig. 4). Final data on $\Lambda$ and $\bar{\Lambda}$ are needed in order to clarify the situation ; we shall come back to this point in section 6 . The net $\Lambda$ rapidity distribution in central $S S$ collisions is shown in Fig. 6. The corresponding results for minimum bias $p A$ collisions are given in Figs. 7 and 8. It is seen that the normalization of the experimental data is larger than the theoretical one especially for $p-\bar{p}$. Note, however, that by integrating over $y$ the experimental distributions one realizes that their normalization is larger than the number of wounded nucleus 
in $p A u$ (given by the Glauber model) by more than a factor 2. As pointed out in (2) this excess may be due to recoil nucleons which are not completely disentangled from the wounded ones. This point needs clarification.

Note that, in our model, the relative yields $I_{3}$ (eq. (đ)) should apply at higher energies when the phase space limitations are less important. With $S / L=0.3$, they would give a ratio $\Xi^{+}+\Xi^{0} / \Lambda+\Sigma \sim 0.3$ which is in agreement with Fermilab [24] and SPS collider data [25].

\section{Antibaryon production}

In string models, $B \bar{B}$ pair production takes place via diquark-antidiquark pair production in the string fragmentation. It turns out that at present CERN energies only strings of type $q q-q$ have large enough invariant mass to produce $B \bar{B}$ pairs. This gives rise to a scaling of $\bar{B}$ yields in the number of participants. (The number of $q q-q$ strings is proportional to the number of wounded nucleons). Experimentally, the observed increase is much faster - closer to a scaling in the number of collisions. In order to solve this problem it was proposed some time ago [20, 26, 27] to consider the production of $B \bar{B}$ pairs from diquark-antidiquark pairs in the sea of the participating nucleons . The rapidity distribution of antibaryons in $A A$ collisions is then given by

$$
\frac{d N^{A A \rightarrow \bar{B}}}{d y}(y)=\bar{n}_{A}\left(\frac{d N_{\text {string }}^{\bar{B}}}{d y}(y)\right)_{\bar{n} / \bar{n}_{A}}+\left(\bar{n}-\bar{n}_{A}\right)\left(\frac{d N_{\text {sea }}^{\bar{B}}}{d y}(y)\right)_{\bar{n} / \bar{n}_{A}} .
$$

The first term represents the conventional pair production in the string breaking process. As discussed above, it is proportional to the number of participants. The second term corresponds to pair production from a string having a sea diquark or antidiquark at one of their ends. In DPM, the total number of strings is proportional to $\bar{n}$. Since the number of strings with a valence diquark at one of their ends is

\footnotetext{
${ } \mathrm{A}$ different mechanism based on string junction-antijunction exchange has been proposed recently 15.
} 
proportional to $\bar{n}_{A}$, the number of strings with a sea diquark at one of their ends is proportional to $\bar{n}-\bar{n}_{A}$. Of course, pulling a diquark-antidiquark pair out the nucleon sea is dynamically suppressed - in the same way as its production in the string breaking process is suppressed as compared to $q-\bar{q}$ production. Thus, we expect that in each individual string, the production of $B \bar{B}$ pairs in the two components (sea and string) in eq. (7) are comparable. In practice, the normalization of the second component is treated as a free parameter. However, it turns out that the sea component is always smaller than the string one, not only at $y^{*}=0$ (see Table 1 ) but also after integration over rapidity. Note that the string with sea diquarks have a smaller invariant mass.

For the string term we use the results of ref. [20]. The absolute normalization of this term was determined from a fit of the $p p$ data. For the $y$-dependence of the sea term, we also use the results of ref. [20]. As discussed above, its absolute normalization is a free parameter. This parameter is the same for all species of baryons. More precisely, since the baryons and antibaryons in the sea component are made out of three sea quarks or antiquarks, the relative yields of the different baryon species is again given by eq. (41) ${ }^{\text {k* }}$. (We neglect here the small differences in the rapidity shapes induced by the different baryon masses). We are left in this way with a single free parameter for this new sea component. Therefore, we have a total number of two free parameters, one in the diquark breaking component and one in the sea component - plus the value of the strangeness suppression factor $S / L$ for which two values (0.22 and 0.3$)$ have been considered. Of course, the conventional components $D P$ and string in eqs. (2) and (7) contain several free parameters. However, as discussed above, these parameters have been fixed in ref. [20] from a fit of the $p p$ data and are not changed here $\square$. The values at $y^{*}=0$ of the various

\footnotetext{
${ }^{* *}$ Note that in this case $\alpha=1$, i.e. no admixture of the type discussed in connection with the $D B$ component is present here.

${ }^{\dagger \dagger}$ For this reason, the change in the strange suppression parameter $S / L$ from 0.22 to 0.3 only applies to the new components $D B$ and sea in eqs. (2) and (1) (see Table 1).
} 
components for the different baryon species are given in Table 1.

The results for the rapidity distributions of the $\Lambda+\bar{\Lambda}, \Xi^{-}+\Xi^{+}, \Xi^{-}$and $\Omega+\bar{\Omega}$ yields in central $\mathrm{Pb}-\mathrm{Pb}$ collisions at $158 \mathrm{GeV}$ are given in Figs. 9-12. The $p, \bar{p}$, $Y$, and $\bar{Y}$ yields at $\left|y^{*}\right|<0.5$ in minimum bias $p P b$ collisions as well as at four different centralities in $\mathrm{Pb}-\mathrm{Pb}$ collisions, are given in Fig. 13. The corresponding ratios $R_{Y}=\bar{Y} / Y$ at $\left|y^{*}\right|<0.5$ are given in Fig. 14. It should be noted that the values of $R_{Y}$ are not absolute predictions of our model. They can be changed by changing the normalization of the sea component in eq. (7). However, the ratios $R_{p}: R_{\Lambda}: R_{\Xi}: R_{\Omega}$ are a characteristic feature of the model. They show an increase with the number of strange quarks in the baryon. In Fig. 15 we show the various baryon yields at $y^{*}=0$ divided to the average number of participants, $2 \bar{n}_{A}$, normalized to the same quantity in $p P b$. A discussion of these results is given in Section 6, after introducing final state interaction.

\section{$5 \quad$ Final state interaction}

In an attempt to explain the strong enhancement of the $\Omega+\bar{\Omega}$ yield observed by the WA97 collaboration [14], we are going to use our results for the baryon densities as initial conditions in the gain and loss differential equations which govern final state interactions [17, 28]

$$
\frac{d N_{i}}{d^{4} x}=\sum_{k, \ell} \sigma_{k \ell} \rho_{k}(x) \rho_{\ell}(x)-\sum_{k} \sigma_{i k} \rho_{i}(x) \rho_{k}(x)
$$

The first term in the r.h.s. of (\$) describes the production of particles of type $i$ resulting from the interaction of particles $k$ and $\ell$ with space-time densities $\rho(x)$ and cross-sections $\sigma_{k \ell}$ (averaged over the momentum distribution of the interacting particles). The second term describes the loss of particles of type $i$ due to its interaction with particles of type $k$. We use cylindrical space-time variables and assume boost invariance (i.e. the densities $\rho(x)$ are taken to be independent of $y$ ). 
If we furthermore assume that the dilution in time of the densities is mainly due to longitudinal motion, i.e. :

$$
\rho_{i}(\tau, y, \vec{s})=\rho_{i}(\tau, \vec{s}) \frac{\tau_{0}}{\tau}
$$

where $\tau=\sqrt{t^{2}-z^{2}}$ is the proper time and $\vec{s}$ the transverse coordinate, eqs. (8) can be written as 28]

$$
\tau \frac{d \rho_{i}}{d \tau}=\sum_{k, l} \sigma_{k \ell} \rho_{k} \rho_{\ell}-\sum_{k} \sigma_{i k} \rho_{i} \rho_{k} .
$$

Here $\rho_{i}(y, \vec{s}, \vec{b})=d N_{i} / d y d \vec{s} d \vec{b}$. Thus, at fixed impact parameter $\vec{b}$, we have to know the rapidity densities per unit of transverse area $d \vec{s}$. Our eqs. (2) and (7) do give these rapidity densities - the dependence on $\vec{s}$ and $\vec{b}$ is contained in the geometrical factors $\bar{n}_{A}$ and $\bar{n}$, given by the Glauber model. In the following, we use nuclear profiles obtained from Saxon-Woods nuclear densities using the three-parameter Fermi distribution of ref. [29]. For the pion densities we use the DPM results of ref. [30 - where explicit expressions as a function of $\bar{n}_{A}$ and $\bar{n}$ are given.

Eqs. (10) have to be integrated from initial time $\tau_{0}$ to freeze-out time $\tau$. These equations are invariant under the change $\tau \rightarrow c \tau$. Therefore the result depends only on the ratio $\tau / \tau_{0}$. Following refs. [30, 31], we use the (inverse) proportionality between $\tau$ and $\rho$ and put $\tau / \tau_{0}=\rho(y, \vec{s}, \vec{b}) / \rho_{f_{0}}$. Here $\rho(y, \vec{s}, \vec{b})$ are the initial densities given by our expressions obtained in previous sections and $\rho_{f_{0}}$ is the freeze-out density. For the latter, we take the charged density per unit rapidity in a $p p$ collision, i.e. $\rho_{f_{0}}=\left[3 / \pi R_{p}^{2}\right]\left(d N^{-} / d y\right)_{y^{*}=0}=1.15 \mathrm{fm}^{-2}$ [30, 31].

We have now to specify the channels that have been taken into account in our calculation. They are :

$$
\pi N \rightarrow K \Lambda, \quad \pi N \rightarrow K \Sigma, \quad \pi \Lambda \rightarrow K \Xi, \quad \pi \Sigma \rightarrow K \Xi, \quad \pi \Xi \rightarrow K \Omega
$$


and the corresponding reactions for antiparticles. To be more precise, of all possible charge combinations in (11), some are of the type shown in Fig. 16a, with annihilation of a light quark pair and production of an $s-\bar{s}$. They have all been taken into account with the same cross-section $\sigma=0.14 \mathrm{mb}$. All other reactions in (11) are of the type shown in Fig. 16b. They have three quark lines in the $t$-channel (baryon exchange). Their average cross-section are smaller than the one of Fig. 16a and have been neglected $\oplus$. We have also neglected all strangeness exchange reactions (Fig. 16c) $K N \longleftrightarrow \pi \Lambda$ etc. Although the corresponding cross-sections are larger at threshold, this is no longer the case for the cross-sections averaged over the momentum distributions of the interacting particles. (This is due to their steep decrease with increasing energy (see [17). Channels (11) are thus dominant due to the relations $\rho_{N}>\rho_{\Lambda}>\rho_{\Xi}>\rho_{\Omega}$ and $\rho_{\pi}>\rho_{K}$ between particle densities. The results, obtained after solving numerically eqs. (10), with our initial densities and a common value of the averaged cross-section $\sigma=0.14 \mathrm{mb}$ for all channels, are shown in our figures by a full line in the case $S / L=0.3$ and $\alpha=0.23$ and by a dashed-dotted line in the case $S / L=0.22$ and $\alpha=0.5$. (A comparable value of $\sigma$ has been obtained in ref. [17] in a hadron gas model).

The effect of the final state interaction is negligeably small in $p A$ collisions. In central $S S$ collisions its effect on the $p$ and $\Lambda$ yields is very small (less than $5 \%$ ). The effect increases with the number of strange quarks in the produced hyperon. In central $\mathrm{Pb}-\mathrm{Pb}$ collisions, with our value of the cross-section, it turns out to be comparatively small for $p$ and $\Lambda$ yields. However, it increases the $\Xi$ yields by up to $50 \%$ and the $\Omega+\bar{\Omega}$ yield by a factor 5 . Agreement with the WA97 data [14 is obtained in this way (Fig. 13).

\footnotetext{
市The reactions we have kept are : $\pi^{+}+n \rightarrow K^{+} \Lambda, \pi^{-} p \rightarrow K^{0} \Lambda, \pi^{-}+n \rightarrow K^{0} \Sigma^{-}, \pi^{+} p \rightarrow$ $K^{+} \Sigma^{+}, \pi^{-} \Lambda \rightarrow K^{0} \Xi^{-}, \pi^{+} \Lambda \rightarrow K^{+} \Xi^{0}, \pi^{+} \Sigma^{-} \rightarrow K^{+} \Xi^{-}, \pi^{-} \Sigma^{+} \rightarrow K^{0} \Xi^{0}, \pi^{-} \Xi^{0} \rightarrow K^{0} \Omega$ and $\pi^{+} \Xi^{-} \rightarrow K^{+} \Omega$ for the reactions initiated by $\pi^{+}$or $\pi^{-}$. For all of them, as well as for the corresponding ones with antiparticles, we take $\sigma=0.14 \mathrm{mb}$. The reactions initiated by $\pi^{0}$ are either of the type of Fig. 16a or Fig. 16b, depending on whether the $u \bar{u}$ or $d \bar{d}$ component of the $\pi^{0}$ is considered. For this reason all these reactions have been included with cross-section $\sigma / 2$.
} 
It is important to note that, due to the small value of $\sigma$, the final state interaction has an important effect only on very rare processes such as $\Omega$ production. It cannot drive the system into chemical equilibrium - even locally.

\section{Discussion}

We discuss here the main features of our results. For $\Lambda+\bar{\Lambda}$ our results for $P b-P b$ are slightly higher than the WA97 data and grossely underestimate NA49 ones at mid-rapidities (Fig. 9). Note, however, that the latter are very preliminary and are currently under reanalysis. For central $S S$ collisions, where the NA35 data are final, we slightly underestimate their net $\Lambda$ yield and slightly overestimate the total $\Lambda$ yield form NA36 [32] (Fig. 6). However, the NA35 value for the $\bar{\Lambda}$ yield at mid rapidities $0.75 \pm 0.15$ is about two times larger than our result. Note that this experimental point looks "anomalous": compared with the WA97 value for the most central rapidity bin in $P b-P b$ collisions, $(1.8 \pm 0.2)$, there is an increase by a factor 2.4 - whereas the number of participants increases by a factor 7 . Note also that NA35 finds a ratio $\bar{\Lambda} / \bar{p}=1.9 \pm 0.7$ at mid-rapidities, while in our model this ratio is always smaller than one (see Fig. 13). This important point needs clarification. In particular, final values of this ratio in $\mathrm{Pb}-\mathrm{Pb}$ collisions are needed.

Concerning, the cascade yields, the NA49 data are published [13]. They are $30 \div 40 \%$ higher than the WA97 ones 14 at mid rapidities. Our results, after final state interaction, are intermediate between the two sets of data - but somewhat closer to the NA49 results (Figs. 10 and 11). The $\Omega+\bar{\Omega}$ yields are in agreement with the WA97 data, after final state interaction (Fig. 12 and 13).

As discussed in Section 4, in our model, the ratios $R_{Y}=\bar{Y} / Y$ increase with the number of strange quarks in the baryon (Fig. 14). This tendency is also seen in the data. However, the ratio of ratios $R_{\Xi} / R_{\Lambda}$ is somewhat too small in our model as compared to the WA97 data [14], but agrees with the NA49 ones [1, 13]. (Remember, 
however, that the value of $R_{\Lambda}$ from NA49 is preliminary).

Another characteristic feature of our approach is that, at mid-rapidities, hyperons are more strongly enhanced than antihyperons. As a consequence, the ratio $\bar{Y} / Y$ decreases between $p P b$ and central $P b-P b$ collisions (Fig. 14). This is due to the strong effect of the $D B$ component in the net baryon yield. Final state interaction works in the same direction. This important feature of our results is seen in the data [14].

Finally, the WA97 collaboration has found that the increase of the hyperon and antihyperon yields per participant (Fig. 15) increases faster than the number of participants between $\mathrm{pPb}$ and the first centrality bin in $\mathrm{Pb}-\mathrm{Pb}$. However, between the first and last centrality bin all yields approximately scale with the number of participants. We find an increase which is faster in the first case than in the second one. However, some mild increase is left in $\mathrm{Pb}-\mathrm{Pb}$ (Fig. 15).

\section{Conclusions}

The large baryon stopping observed in central heavy ion collisions at CERN energy is not reproduced by any of the available independent string models - at least in their original form. We have modified the DPM by introducing a new realization of the diquark breaking mechanism. We reproduce in this way the observed net baryon yield. This mechanism also produces an important enhancement of net hyperons. At this level, the new version of DPM presented here (which has also diquarkantiquark pairs in the nucleon sea) remains strictly an independent string model. It reproduces with two free parameters, the observed yields of $p$ and $\Lambda$ and their antiparticles in $p A$ and $P b-P b$ collisions. Cascades in central $P b-P b$ collisions are underestimated by less than $50 \%$ while $\Omega$ 's are too small by a factor 5 . Agreement with experiment is restored by introducing final state interaction with an averaged cross-section as small as $\sigma=0.14 \mathrm{mb}$. In this way, we depart from string indepen- 
dence. However, with this small value of the cross-section, there is no significant effect on the bulk of particle production. A comparable value of the cross-section for final state interaction was obtained in ref. [17] from the experimental data on the energy dependence of cross-sections, averaged over the momentum distribution of the interacting particles obtained in the hadron gas model. The smallness of this averaged cross-section lead the authors of [17] to argue that strangeness phase space saturation would be too slow in a hadron gas. It is interesting that such a small value of the averaged cross-section allows to reproduce the observed enhancement of multi-strange hyperons and antihyperons in central $\mathrm{Pb}-\mathrm{Pb}$ collisions.

The main features of our results are the following : 1) The hyperon yields per participant increase faster than antihyperon ones. As a consequence, the ratio $R_{Y}=\bar{Y} / Y$ decreases between $p P b$ and central $P b-P b$ collisions ; 2) The ratios $R_{Y}$ increase with the number of strange quarks in the hyperon ; 3) The increase of the $Y$ and $\bar{Y}$ yields per participant is faster between $p P b$ and the first centrality bin in $\mathrm{Pb}-\mathrm{Pb}$ collisions and slows down between the first and last centrality bins of WA97. All these features are also present in the data.

\section{Acknowledgments}

It is a pleasure to thank N. Armesto, A. Casado, E. G. Ferreiro, A. B. Kaidalov, C. Pajares and J. Tran Thanh Van for discussions. We also thank R. Lietava, P. Seyboth and O. Villalobos Baillie for information on the data. A.C. acknowledges partial support from a NATO grant OUTR.LG 971390. C.A.S. thanks Fundación Caixa Galicia from Spain for financial support. 


\section{Figure Captions}

Fig. 1 : Conventional diquark preserving (DP) fragmentation mechanism for net baryon production.

Fig. 2 : Example of diquark breaking (DB) diagram for net baryon production in $p A$ with two inelastic collisions.

Fig. 3 : Rapidity distribution of the net baryon number $(B-\bar{B})$ in central $S S$ (200 AGev/c) and PbPb (158 AGev/c) collisions. The full lines are obtained from eq. (2). The data are from ref $[1,2]$ (for central $S S$ collisions the data are obtained as $B-\bar{B}=2(p-\bar{p})+1.6(\Lambda-\bar{\Lambda}))$. Open circles are data reflected about $y^{*}=0$ (errors not shown). The dotted line is the result obtained without the DB component in the case of central $\mathrm{PbPb}$ collisions. Due to baryon number conservation, these results are not affected by final state interactions.

Fig. 4 : Rapidity distributions for net proton production $(p-\bar{p})$ in central $\mathrm{PbPb}$ collisions at $158 \mathrm{AGev/c}$ compared to the results of ref. [1]. Open circles are data reflected about $y^{*}=0$ (errors not shown). The dashed line is our result without final state interactions with a strangeness suppression factor $S / L=0.3$, and the full line is the corresponding result with final state interactions. The dotted-dashed line corresponds to a suppression factor $S / L=0.22$ and with final state interactions. The dotted line is our result without DB component and without final state interactions.

Fig. 5 : Same as Fig. 4 for $\Lambda-\bar{\Lambda}$. Now the three dotted lines are estimates by the NA49 Collaboration [1]. The experimental point at $y^{*}=0$ is form the WA97 Collaboration [14]. 
Fig. 6 : Same as Fig. 5 for central $S S$ collisions. The data are from NA35 [2] (circles). Also shown, the value of the total $\Lambda$ yield measured by NA36 [32] (squares). The theoretical curves are computed with: $\alpha=0.23, S / L=0.3$ (full line); $\alpha=1, S / L=0.3$ (dashed line); $\alpha=0.23, S / L=0.22$ (dotted line) and $\alpha=1, S / L=0.22$ (dashed-dotted line)

Fig. 7 : Same as Fig. 4 for minimum bias $p A u$ interactions. The experimental data are from ref. [2].

Fig. 8 : Same as Fig. 5 for minimum bias $p A u$ interactions. The experimental data are from ref. [2].

Fig. 9 : Same as Fig. 4 for $\Lambda+\bar{\Lambda}$. The point at $y^{*}=0$ (circle) is from the WA97 Coll. [14]. The squares are from NA49 [1].

Fig. 10 : Same as Fig. 9 for $\Xi^{-}+\bar{\Xi}^{+}$. The NA49 data are from ref [13].

Fig. 11 : Same as Fig. 9 for $\Xi^{-}$. The NA49 data are from ref [13].

Fig. 12 : Same as Fig. 9 for $\Omega+\bar{\Omega}$. The experimental point is from WA97 [14].

Fig. 13 : Yields of $p, \Lambda, \Xi^{-}, \Omega+\bar{\Omega}, \bar{p}, \bar{\Lambda}$ and $\bar{\Xi}^{+}$for minimum bias $p P b(158 \mathrm{Gev} / \mathrm{c})$ and central $\mathrm{PbPb}$ collisions $(158 \mathrm{AGeV} / \mathrm{c})$ in four centrality bins. Experimental data are from WA97 [14] (black points) and NA49 [13] (open square). Full (dashed) lines are our results with (without) final state interactions for strangeness suppression factor $S / L=0.3$. The dashed-dotted lines are our results with final state interactions for $S / L=0.22$.

Fig. 14 : Ratios $\bar{B} / B$ at $y^{*}=0$ for minimum bias $p P b$ and $P b P b$ collisions in four different centrality bins at $158 \mathrm{AGev} / \mathrm{c}$. Black circles, squares and triangle correspond to experimental data of WA97 [14] for $\bar{\Lambda} / \Lambda, \bar{\Xi}^{+} / \Xi^{-}$and $\bar{\Omega} / \Omega$ respectively. Open squares are NA49 data [13]. Full lines are our results 
with final state interactions and dashed lines without final state interactions, both for $S / L=0.3$.

Fig. 15 : Same as Fig. 14 for the baryon yields at $y^{*}=0$ in $P b P b$ divided by the number of participant nucleons relative to the same ratio in minimum bias $p P b$.

Fig. 16a : Quark diagrams for reactions (11) with light quark pair annihilation and $s-\bar{s}$ quark creation.

Fig. 16b : Quark diagram for reactions (11) with three quark exchange in the t-channel

Fig. 16c : Quark diagram for strangeness exchange reactions. 


\section{Table}

\begin{tabular}{|l|c|c|c|c|}
\hline & $p$ & $\Lambda$ & $\Xi^{-}$ & $\Omega$ \\
\hline & & & & \\
$d N_{\text {sea }}^{\bar{B}} / d y$ & $4.80 \times 10^{-3}$ & $2.15 \times 10^{-3}$ & $3.21 \times 10^{-4}$ & $3.20 \times 10^{-5}$ \\
$d N_{D \bar{D}}^{\Delta B} / d y$ & $9.12 \times 10^{-2}$ & $3.00 \times 10^{-2}$ & $2.86 \times 10^{-3}$ & $1.23 \times 10^{-4}$ \\
\hline & & & & \\
\hline$N_{D P}^{\Delta B} / d y$ & $6.90 \times 10^{-2}$ & $1.40 \times 10^{-2}$ & 0 & 0 \\
$d N_{\text {string }}^{\bar{B}} / d y$ & $8.50 \times 10^{-3}$ & $2.26 \times 10^{-3}$ & $1.65 \times 10^{-4}$ & $5.07 \times 10^{-6}$ \\
\hline$N_{\text {sea }}^{\bar{B}} / d y$ & $6.54 \times 10^{-3}$ & $2.43 \times 10^{-3}$ & $2.98 \times 10^{-4}$ & $2.4 \times 10^{-5}$ \\
$d N_{D B}^{\Delta B} / d y$ & $9.56 \times 10^{-2}$ & $2.62 \times 10^{-2}$ & $2.29 \times 10^{-3}$ & $1.23 \times 10^{-4}$ \\
\hline
\end{tabular}

Table 1 : Values of the rapidity densities at $y^{*}=0$ in eqs. (2) and (7) for central $\mathrm{PbPb}$ collisions $\left(\bar{n}_{A}=178, \bar{n}=858\right)$ with $\alpha=0.23$ and $S / L=0.3$ (first four lines) and with $\alpha=0.5$ and $S / L=0.22$ (last two lines). The DP and string contributions are the same in both cases. The value of the DP component for $\Xi^{-}$is not exactly 0, due to the fragmentation mechanism of Fig. 1b. However its value is very small as compared to the other components and has been neglected. 


\section{References}

[1] NA49 collaboration : H. Appelshäuser et al, nucl-exp/98110014.

[2] NA35 collaboration: T. Albert et al, Eur. Phys. J. C2, 643 (1998) ; Z. Phys. C64, 195 (1994).

[3] DPM : A. Capella, U. Sukhatme, C.-I. Tan and J. Tran Thanh Van, Phys. Lett. B81, 68 (1979) ; Phys. Rep. 236, 225 (1994). A. Capella, U. Sukhatme and J. Tran Thanh Van, Z. Phys. C3, 329 (1980). A. Capella and J. Tran Thanh Van, Phys. Lett. B93, 146 (1980) ; Z. Phys. C10, 249 (1981).

[4] QGSM : A. B. Kaidalov, Phys. Lett. B116, 459 (1982); "QCD at $200 \mathrm{TeV",} \mathrm{ed.}$ by L. Ciafarelli and Yu. Dokshitzer, Plenum Press p. 1 (1992). A. B. Kaidalov, K. A. Ter-Martirosyan, Phys. Lett. B117, 247 (1982).

[5] G. C. Rossi and G. Veneziano, Nucl. Phys. B123, 507 (1977).

[6] E. Gotsman and S. Nussinov, Phys. Rev. D22, 624 (1980).

[7] B. Z. Kopeliovich and B. G. Zakharov, Sov. J. Nucl. Phys. 48, 136 (1988) ; Z. Phys. C43, 241 (1989) ; Phys. Lett. B211, 221 (1988).

[8] A. Capella and B. Kopeliovich, Phys. Lett. B381, 325 (1996).

[9] D. Kharzeev, Phys. Lett. B378, 238 (1996).

[10] HIJING : S. E. Vance, M. Gyulassy and X. N. Wang, Phys. Lett B443, 45 (1998).

[11] VENUS : K. Werner, Phys. Rep. 232, 87 (1993). M. Hladik, Ph. D. Université de Nantes, December 1998.

[12] A. Capella, E. G. Ferreiro and C. A. Salgado, Orsay preprint LPT Orsay 99/06, hep-ph/9902232. 
[13] NA49 collaboration : H. Appelshäuser et al, Phys. Lett. B444, 523 (1998).

[14] WA97 collaboration : E. Andersen et al, Phys. Lett. B433, 209 (1998) ; CERNEP/99-29, to be published in Phys. Lett. B.

[15] S. E. Vance and M. Gyulassy, nucl-th/9901009.

[16] A. Capella, Phys. Lett. B387, 400 (1996).

[17] B. Koch, B. Muller and J. Rafelski, Phys. Rep. 142, 167 (1986).

[18] A. B. Kaidalov, Yad. Fiz. 45, 1452 (1987). A. B. Kaidalov and O. I. Piskunova, Z. Phys. C30, 141 (1986).

[19] B. Andersson, G. Gustafson, G. Ingelman and T. Sjöstrand, Phys. Rep. 97, 31 (1983). X. Artru in Phys. Rep. 97, 147 (1983).

[20] A. Capella, A. Kaidalov, A. Kouider-Akil, C. Merino and J. Tran Thanh Van, Z. Phys. C70, 507 (1996).

[21] H1 collaboration : G. Adloff et al, Proc. 29th Inter. Conf. on High-Energy Phys., Vancouver, Canada, July 1998.

[22] B. Kopeliovich and B. Povh, Phys. Lett. B446, 321 (1999).

[23] J. A. Casado, hep-ph/9810357.

[24] E. C. T. Alexopoulos et al, Phys. Rev. D46, 2773 (1992).

[25] UA5 collaboration : G. J. Alner et al, Phys. Rep. 154, 247 (1987).

[26] J. Ranft, A. Capella and J. Tran Thanh Van, Phys. Lett. B320, 346 (1994). H. J. Möhring, J. Ranft, A. Capella and J. Tran Thanh Van, Phys. Rev. D47, 4146 (1993).

[27] A. Capella, Phys. Lett. B364, 175 (1995). 
[28] B. Koch, U. Heinz and J. Pitsut, Phys. Lett. B243, 149 (1990).

[29] C. W. de Jager, H. de Vries and C. de Vries, Atomic Data and Nuclear Data Tables 14, 479 (1974).

[30] N. Armesto and A. Capella, Phys. Lett. B393, 431 (1997) ; N. Armesto, A. Capella and E. G. Fereiro, Phys. Rev. C59, 395 (1999).

[31] D. Kharzeev, C. Lourenço, M. Nardi and H. Satz, Z. Phys. C74, 307 (1997).

[32] NA36 collaboration : E.G. Judd et al, Nucl. Phys. A590, 291 C (1995). 
Figure 1
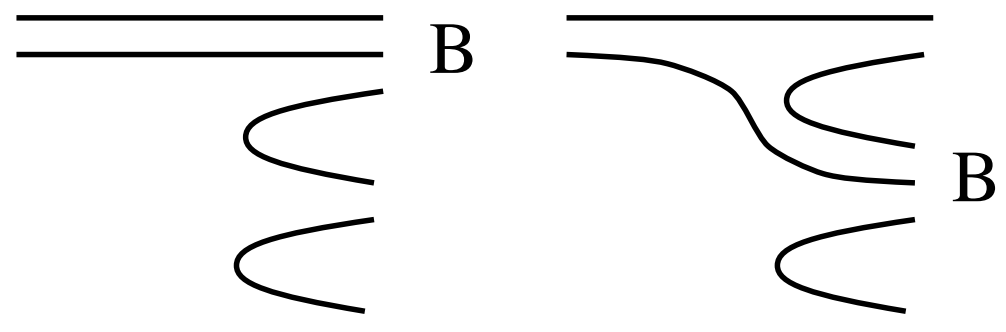

Figure 2

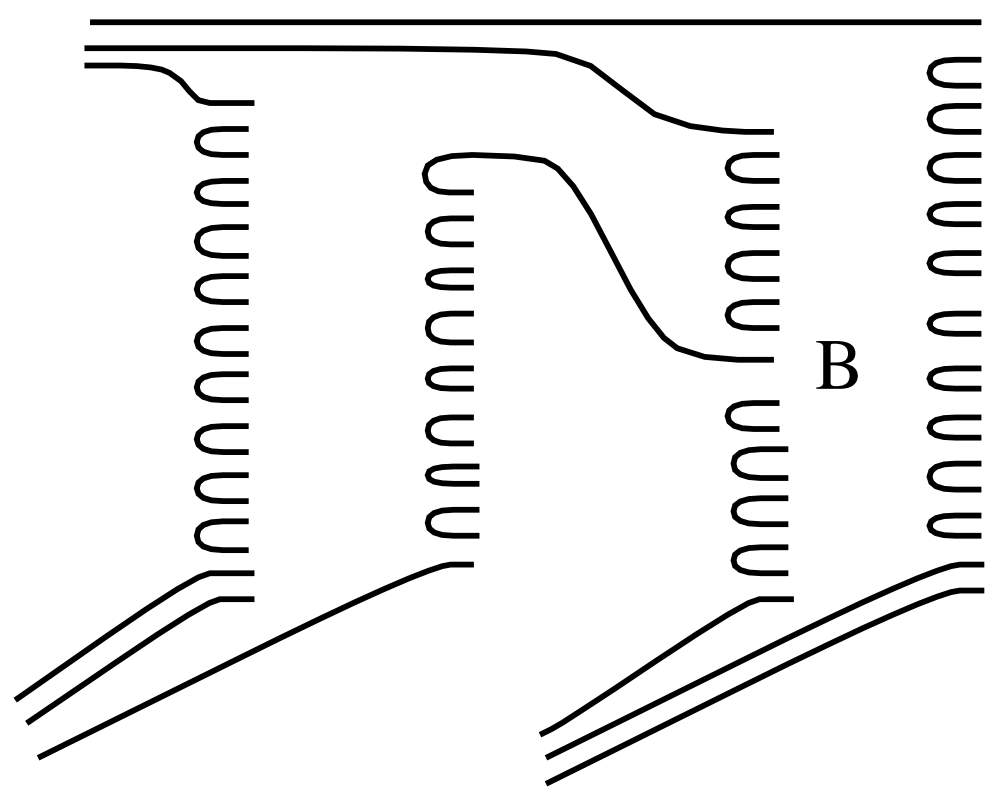


Figure 3

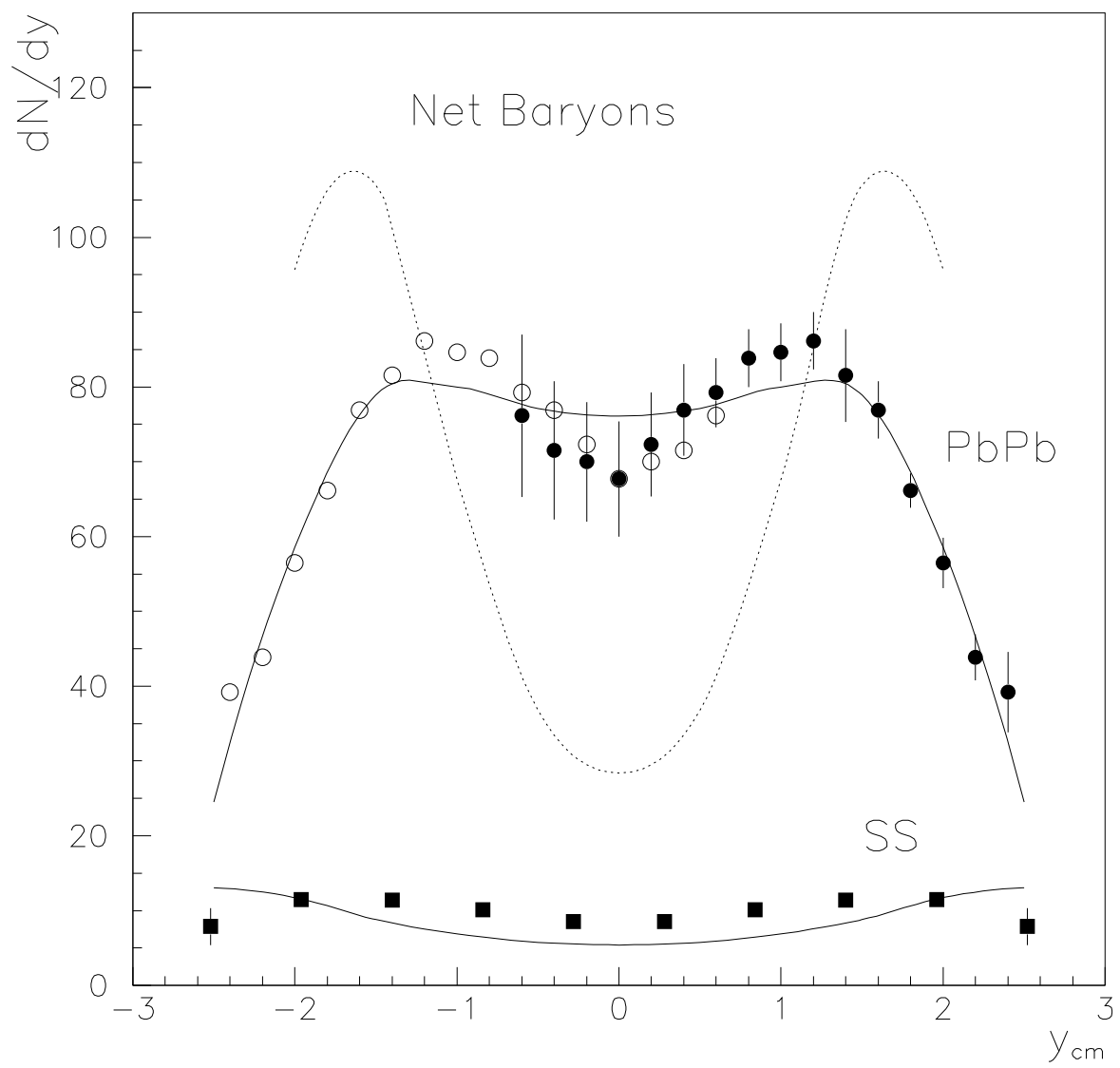


Figure 4

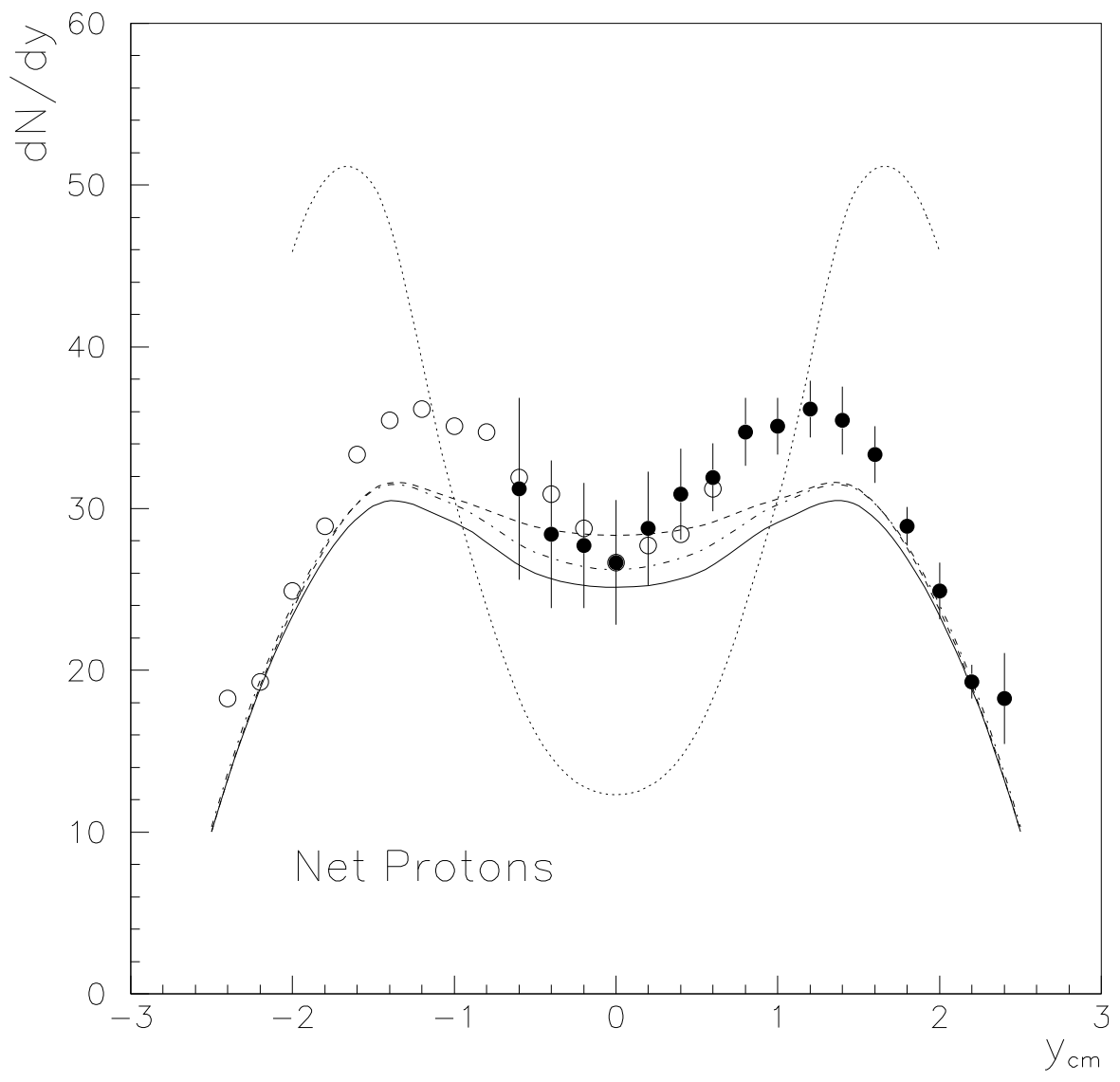


Figure 5

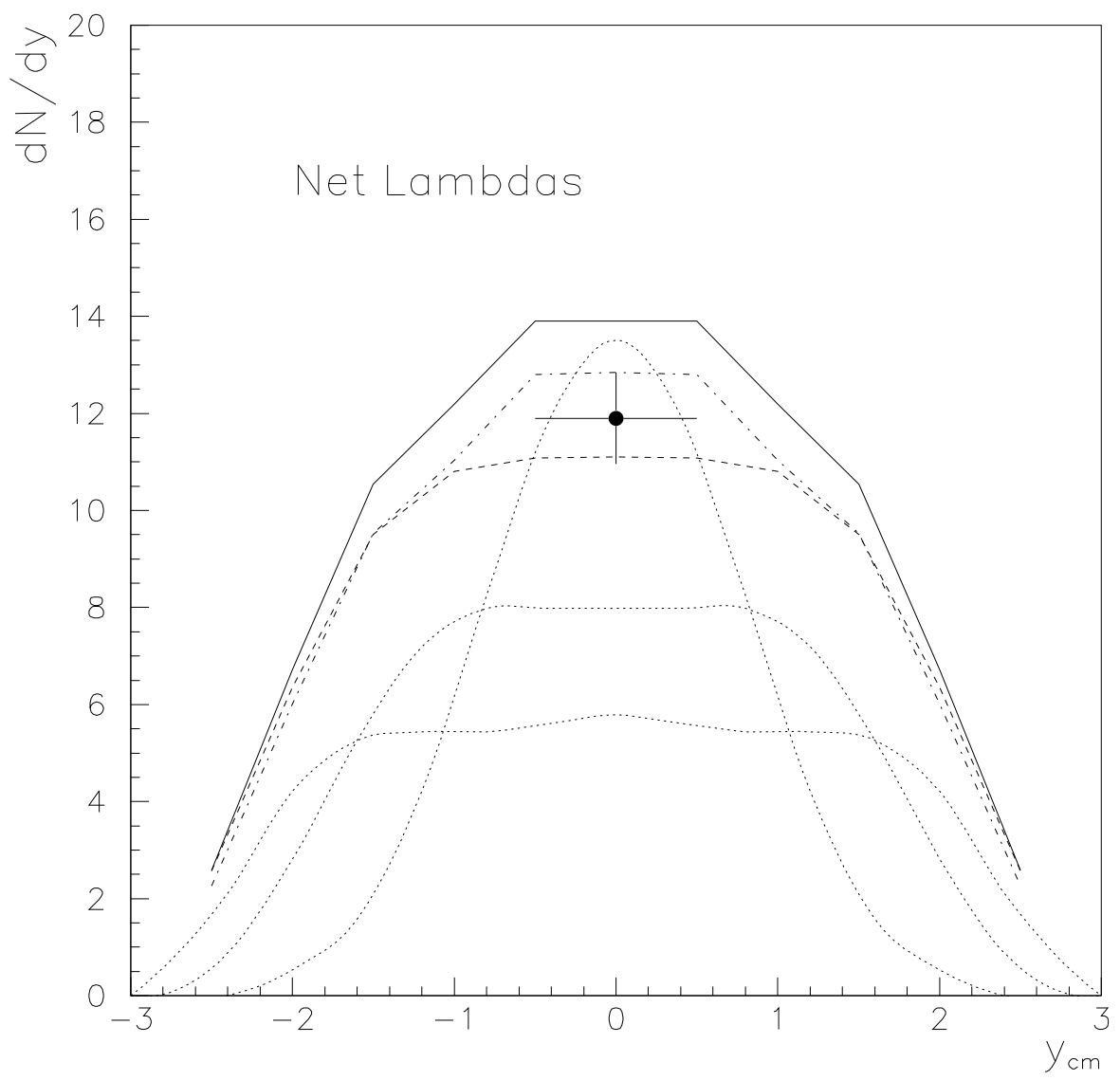


Figure 6

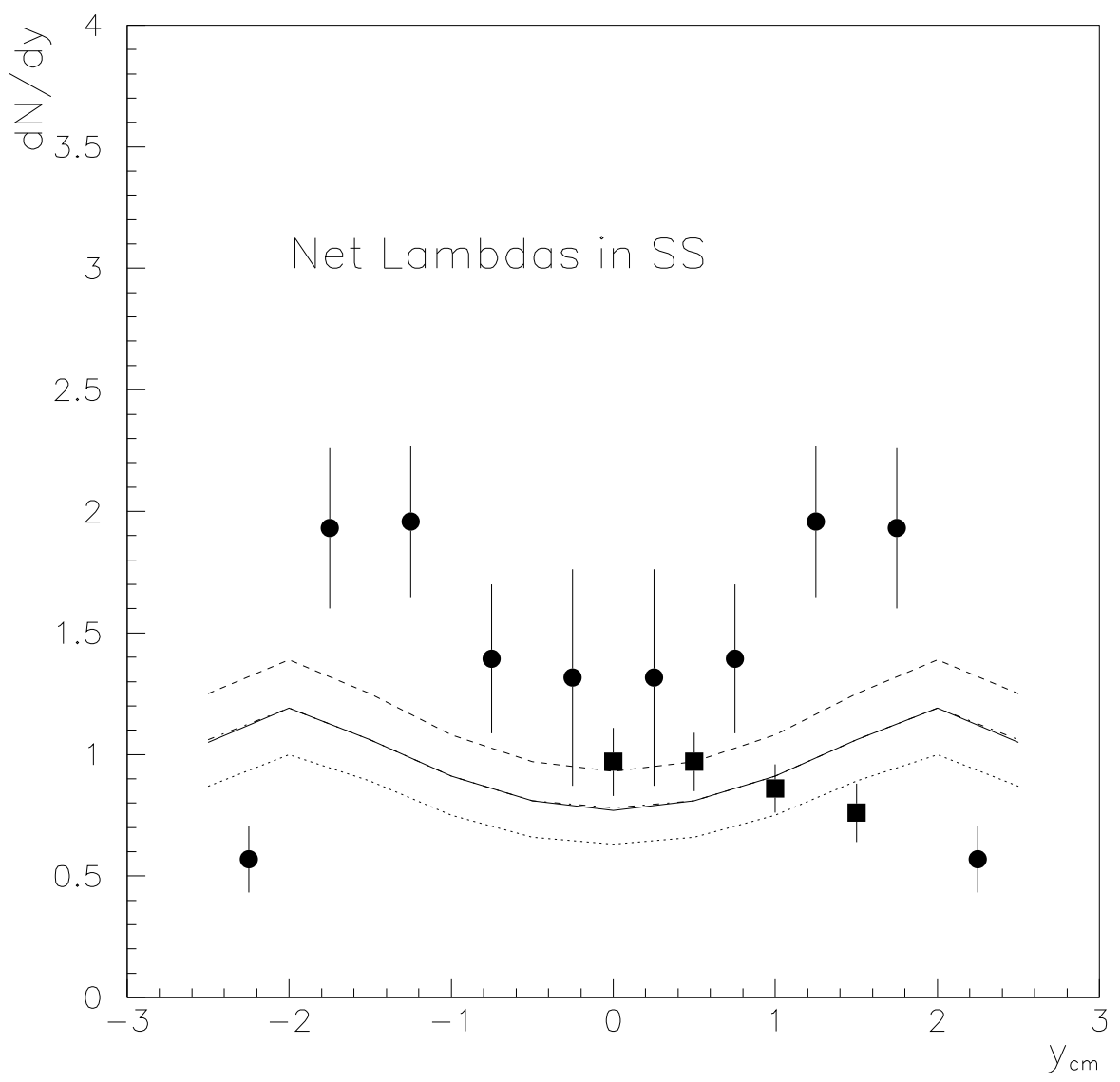


Figure 7

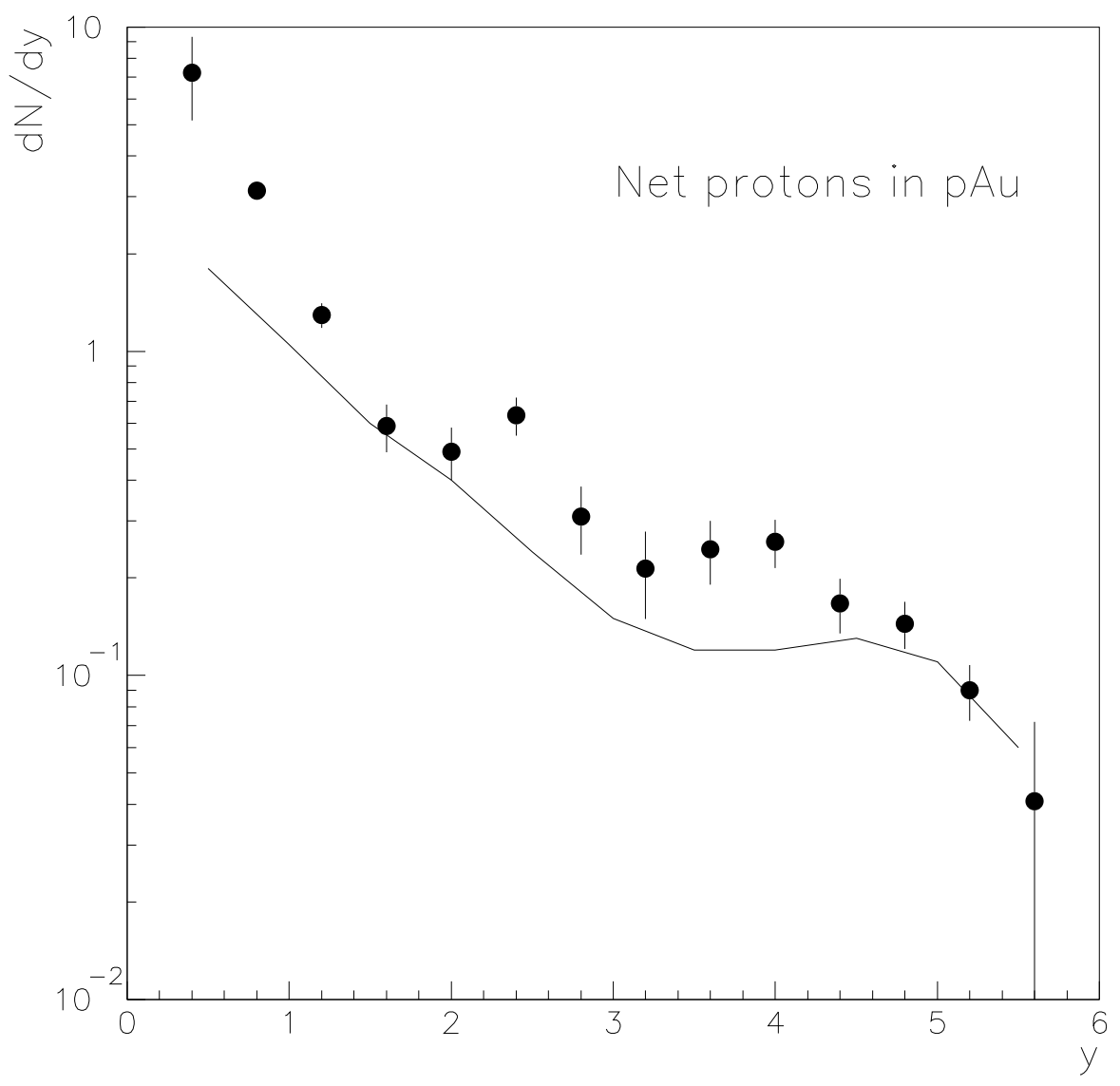


Figure 8

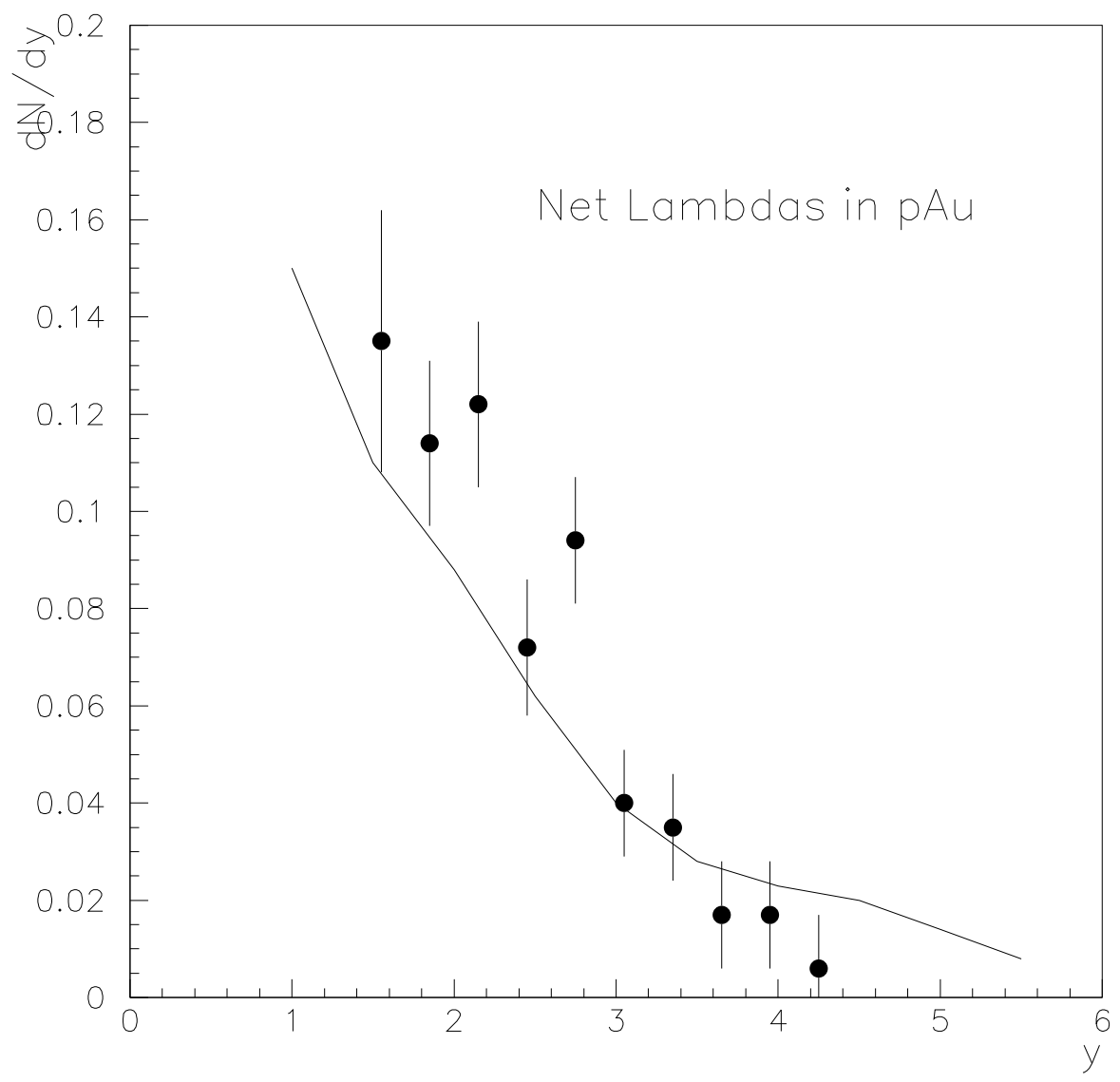


Figure 9

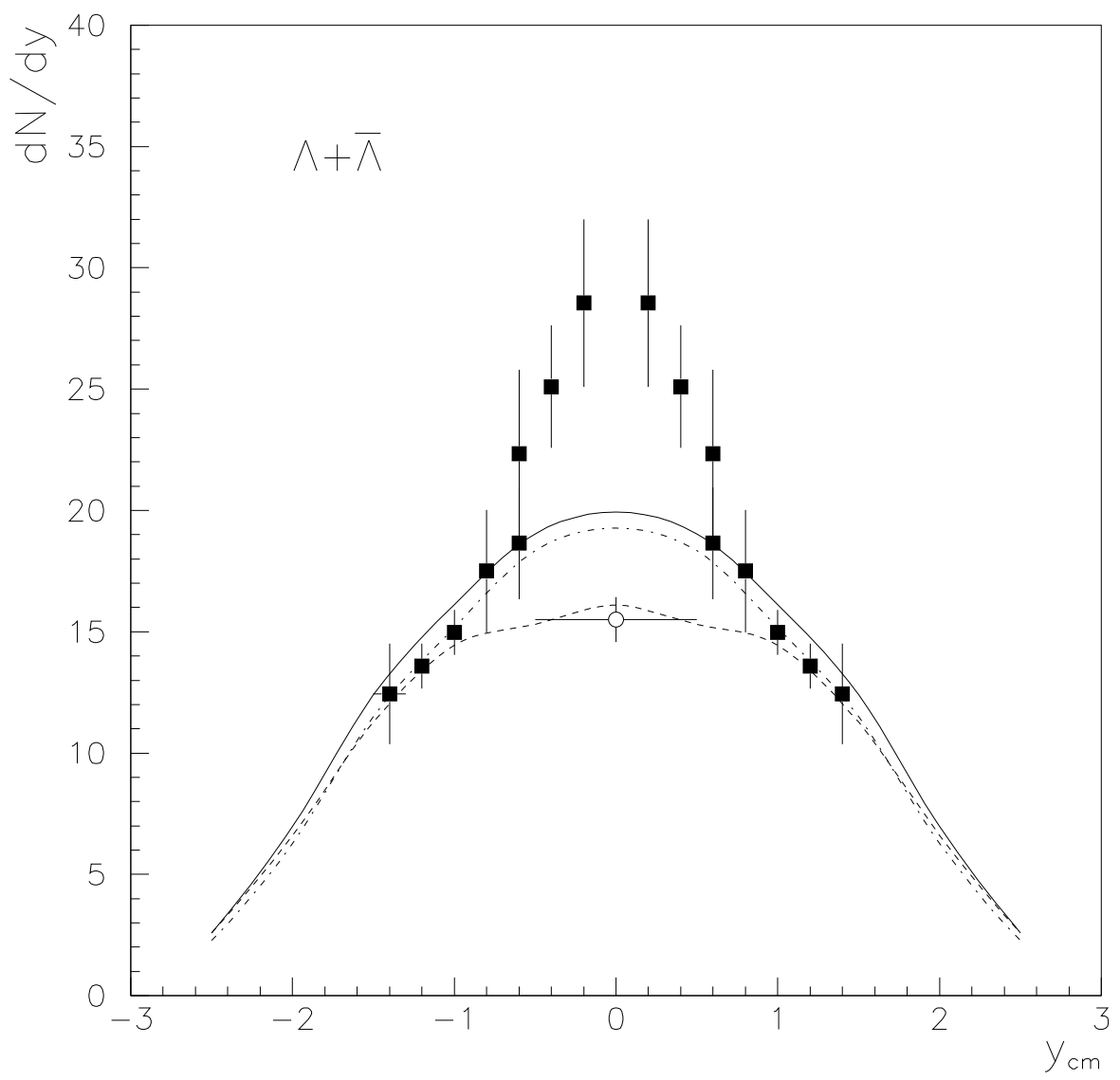


Figure 10

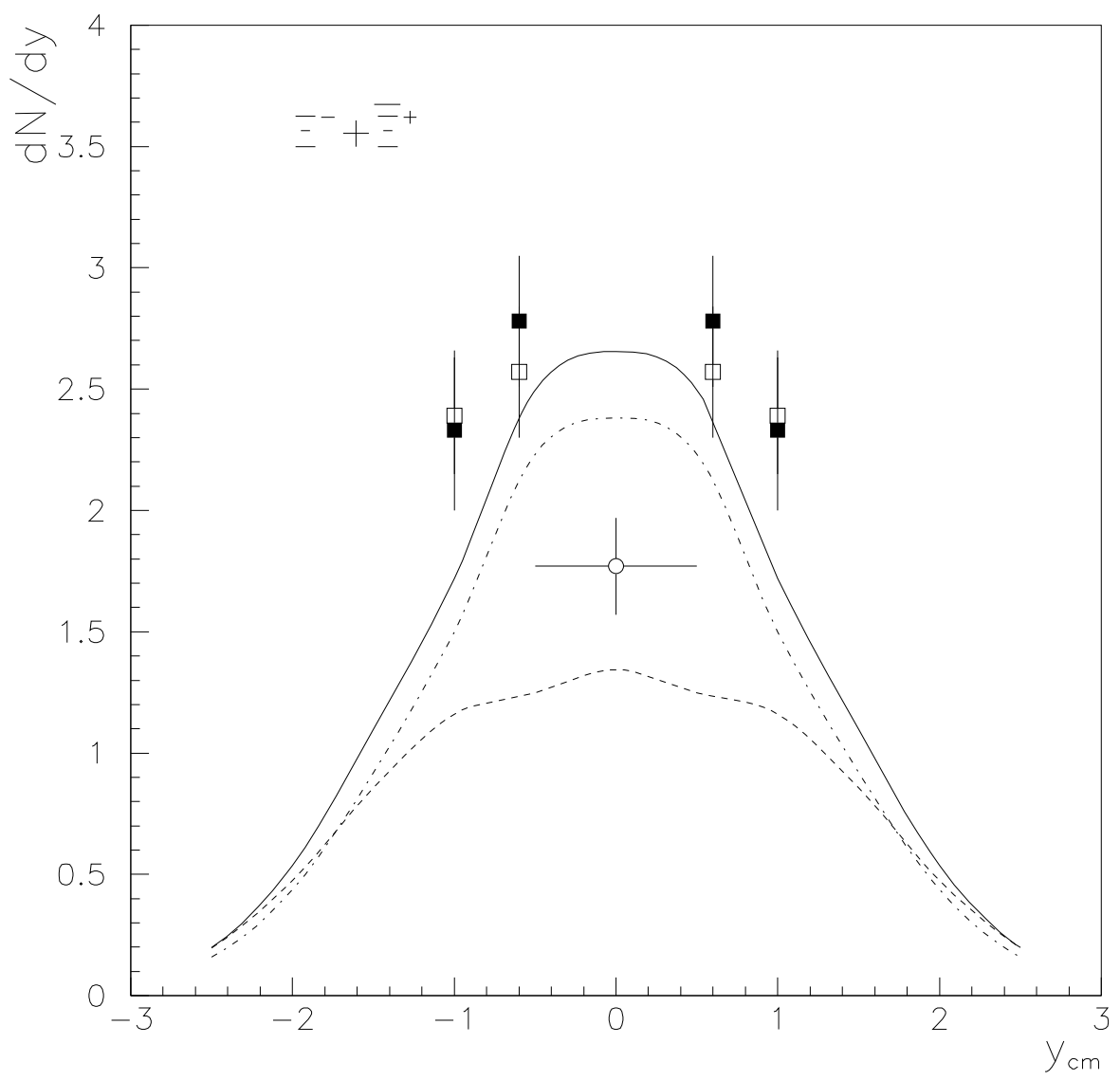


Figure 11

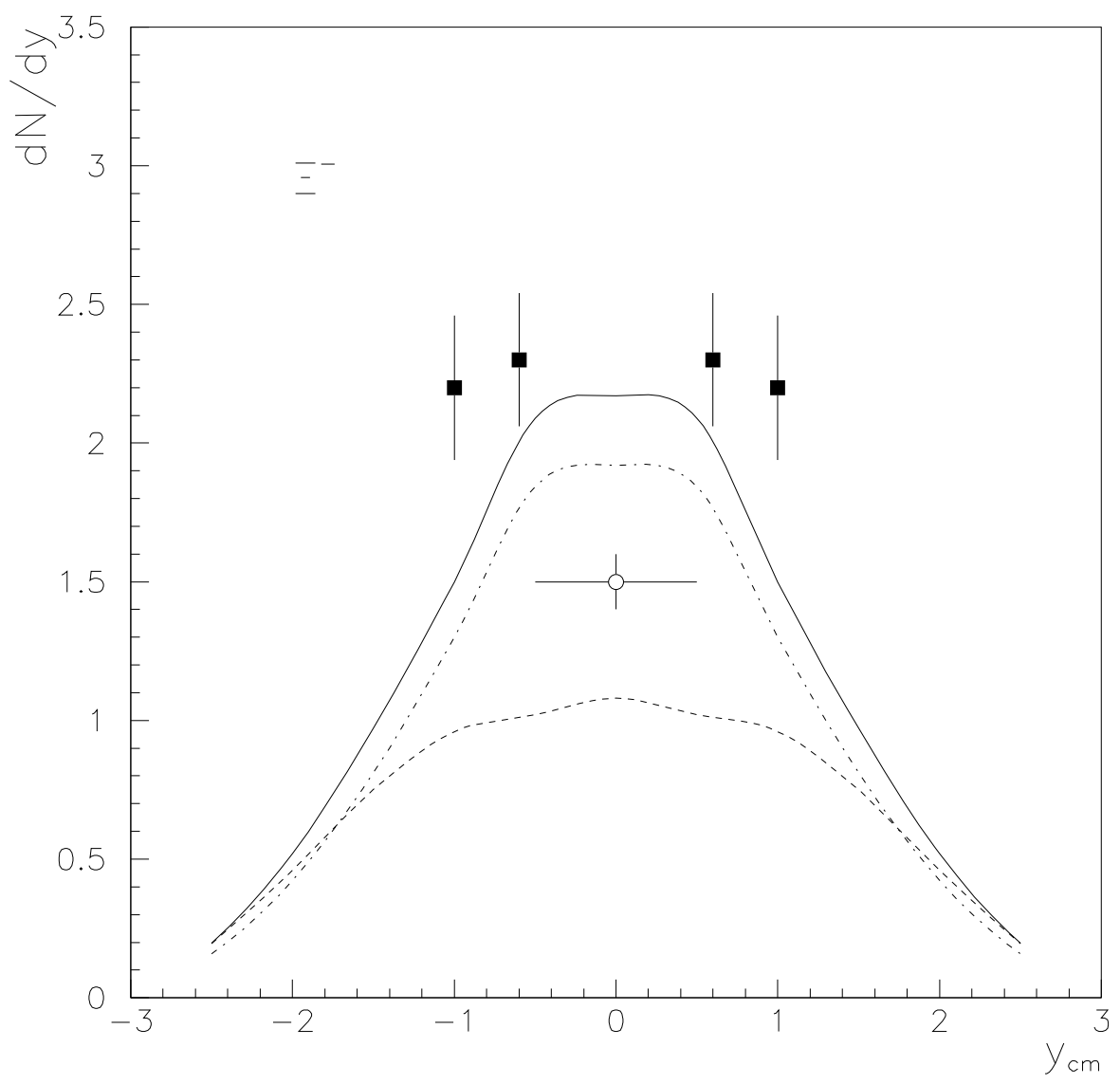


Figure 12

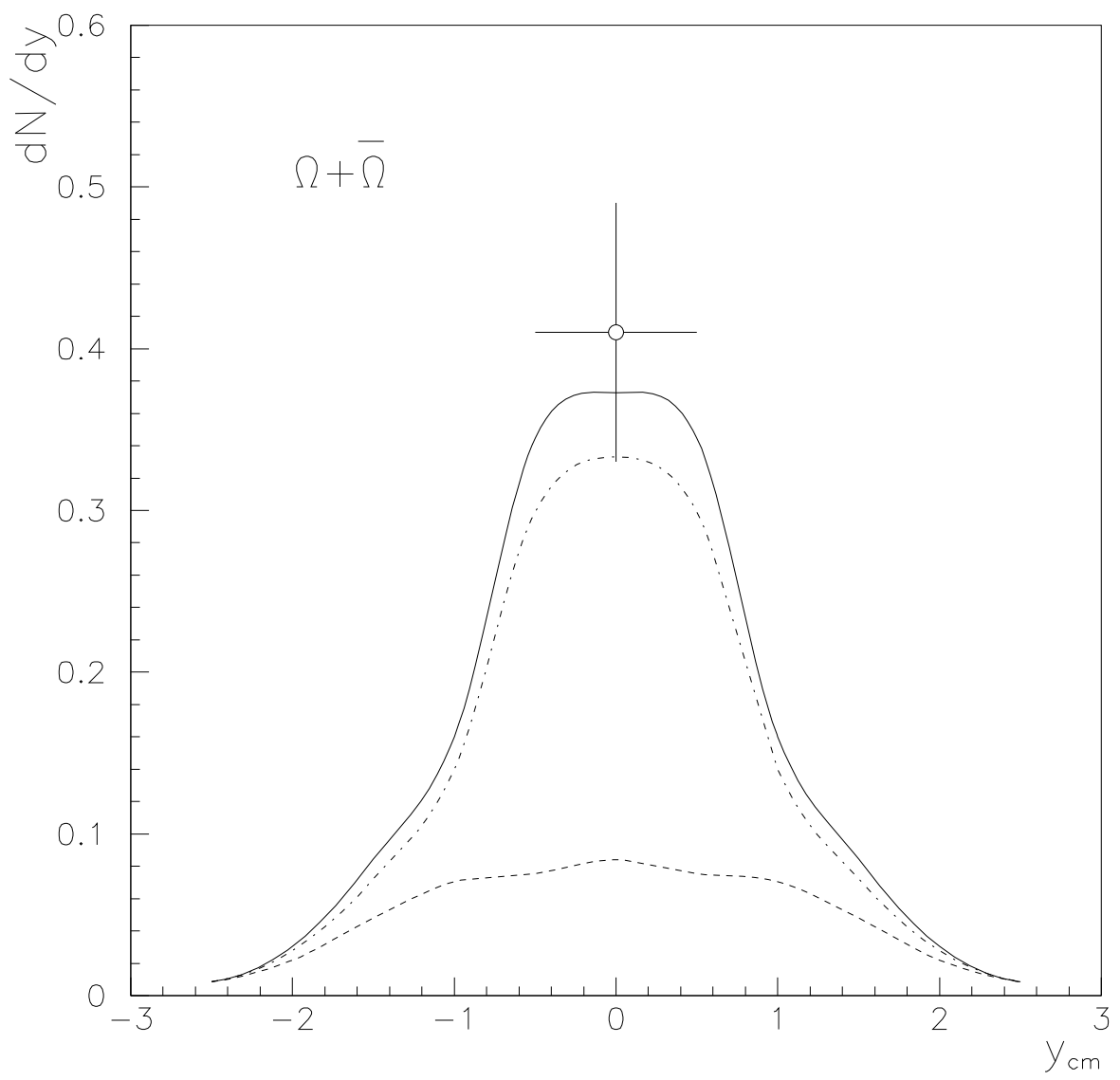


Figure 13

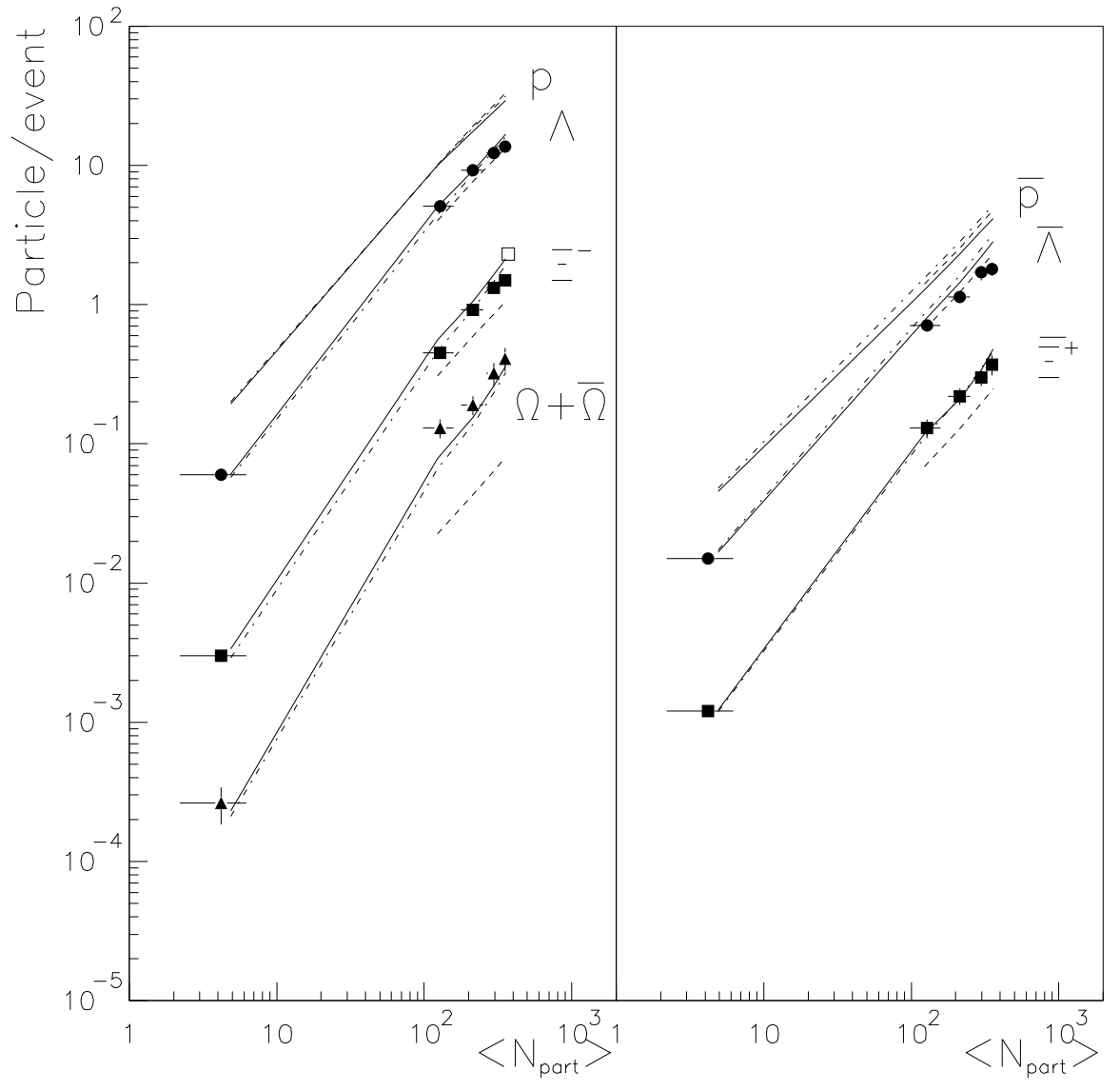


Figure 14

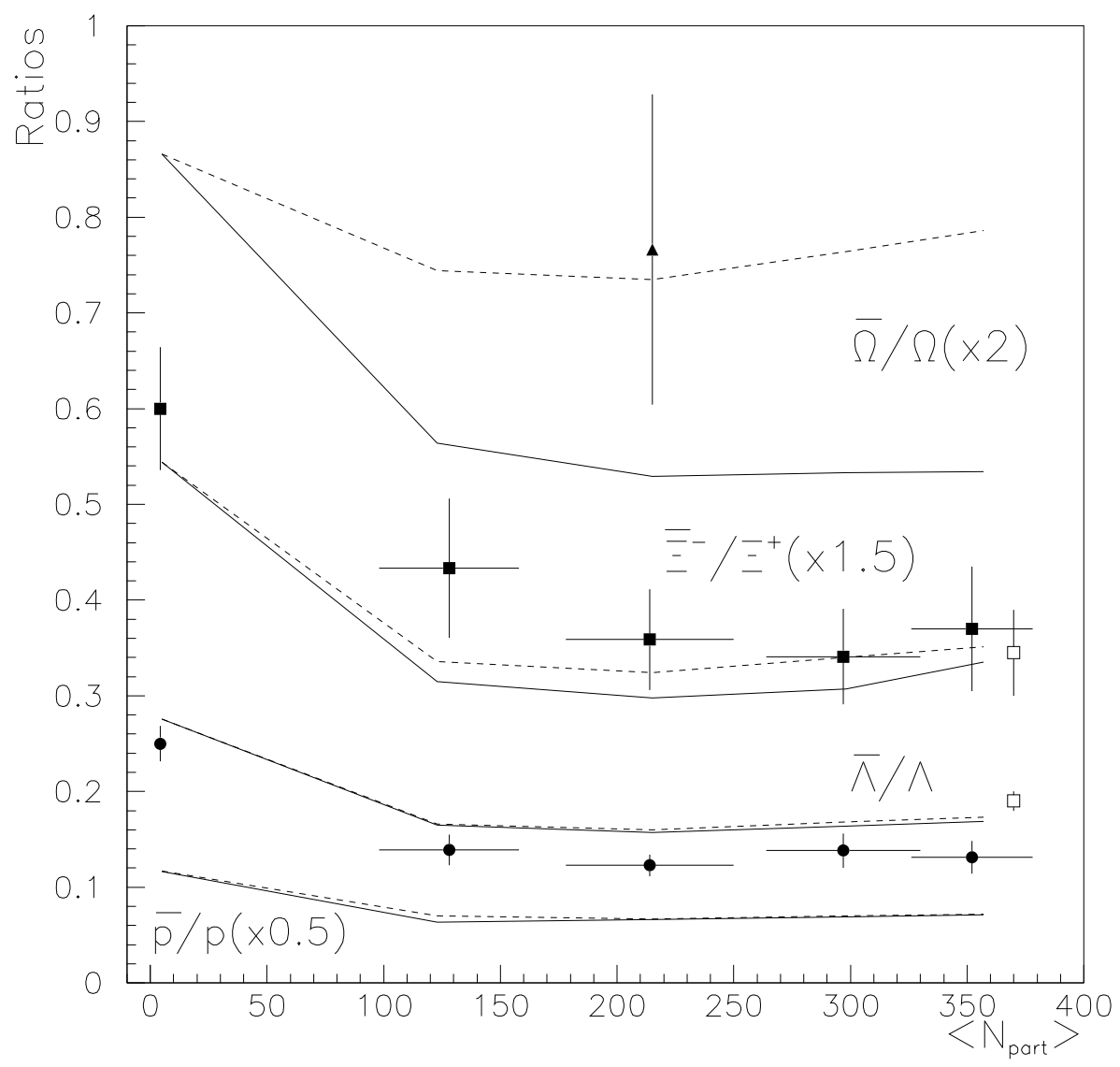


Figure 15

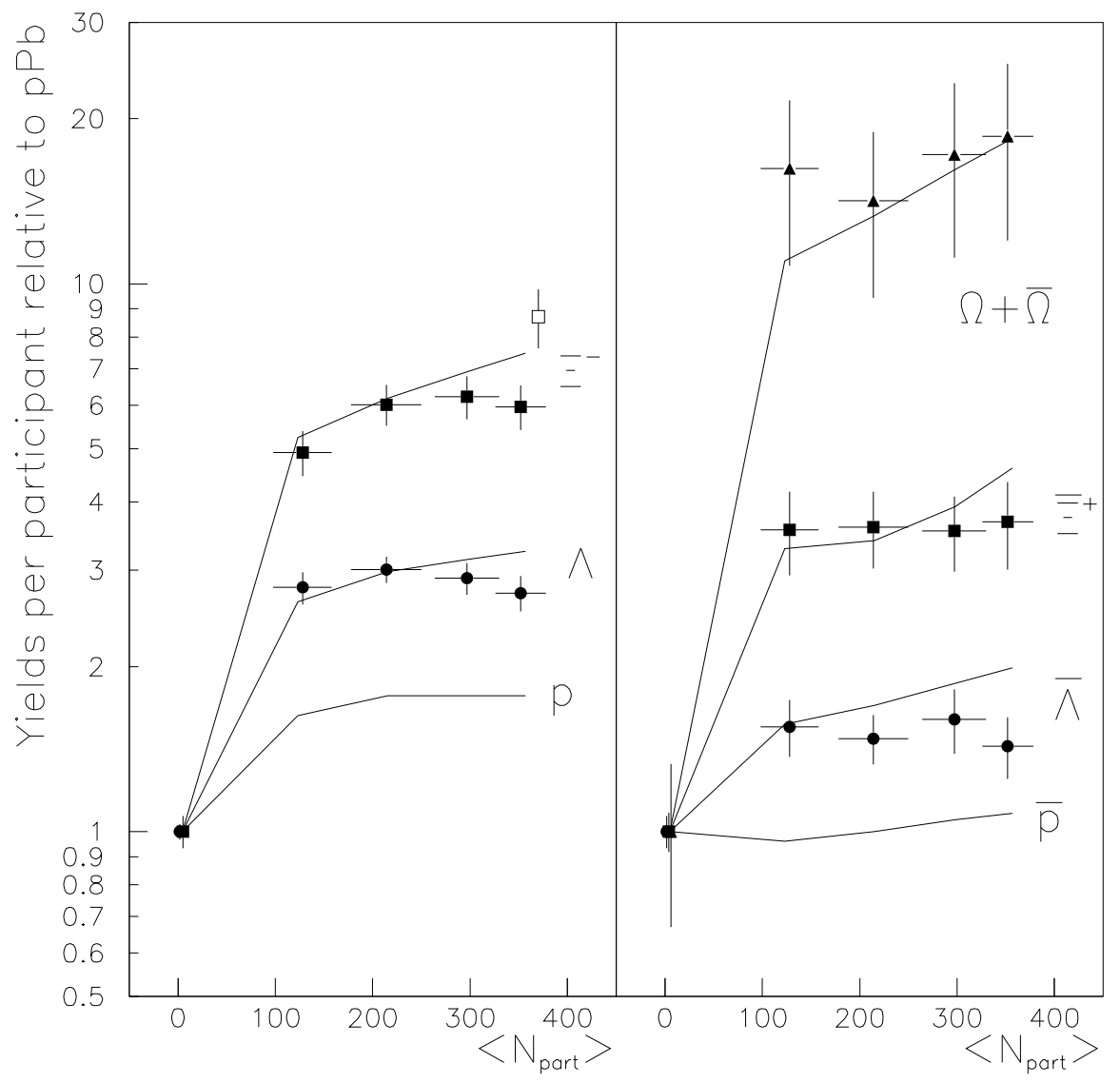


Figure 16a

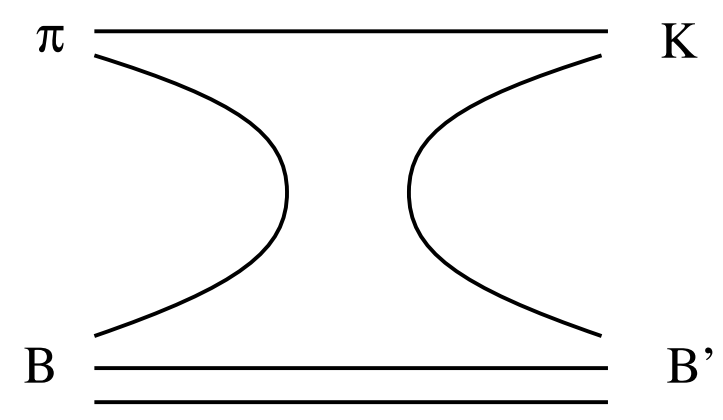

Figure 16b

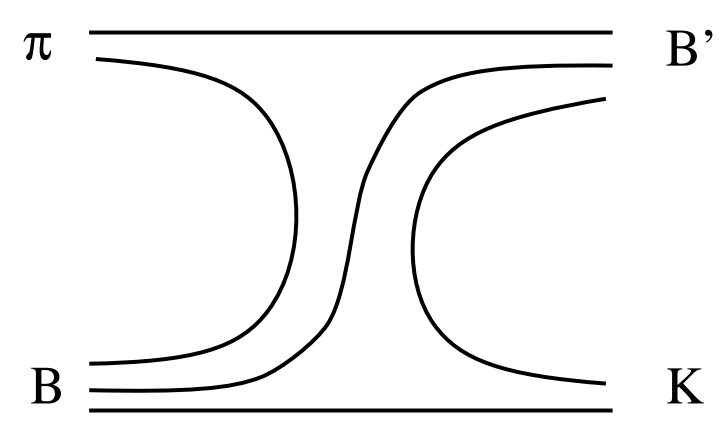

Figure 16c

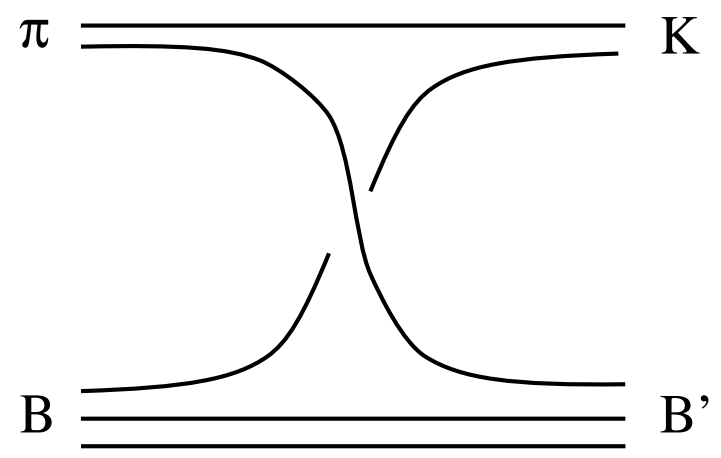

